\title{
Ultrastructure changes and expression of NLRP3 inflammasome in lens anterior capsule of patient with cataracts associated with uveitis
}

Chu Zhang

Anhui Medical University

Chong-Hui Ying

Anhui Medical University

siqin Sun

Anhui Medical University

yuechun Wen

Anhui Medical University

Zicheng Zhu ( $\nabla$ zczhu123@163.com )

Research article

Keywords: Uveitis associated with cataract; lens epithelial cells; NLRP3 inflammasome冈apoptosis

Posted Date: October 17th, 2019

DOI: https://doi.org/10.21203/rs.2.16181/v1

License: (c) (i) This work is licensed under a Creative Commons Attribution 4.0 International License.

Read Full License 


\section{Abstract}

AIM : T o investigate the expression of nod-like receptor pyrin domain 3 (NLRP3) in lens anterior capsule of Uveitis associated with cataract and observe the ultrastructural changes of them .

Methods: 17(22 eyes) cases of uveitis associated with cataract were selected a s experimental group and 10 (18 eyes) cases of age-related cataract were selected a s contro I group. The expressions of NLRP3, apoptosis-related speckle protein $\triangle A S C \otimes$ and caspase- 1 protein were tested by immunohistochemical and the ultrastructural changes of anterior capsul e was observed under electron microscope. - Results: The expression of NLRP3 $₫$ caspase-1 and ASC in the anterior capsu le of cataracts associated with uveitis was significantly higher than that of the control group, the difference was statistically significant ( $p \otimes 0.05)$. The apoptotic changes of lens epithelial cells in uveitis associated with cataract were obvious, and the apoptotic changes of lens epithelial cells were mild in age-related cataract patients. Conclusion: Strongly postive expressed NLRP3 inflamma some and obvious apoptotic changes are founded in the lens epithelial cells of patients with uveitis associat ed with cataract, suggesting that NLRP3 inflamma some and the apoptosis of lens epithelial cells may play a role in the progress of uveitis associat ed with cataract.

\section{Background}

Uveitis associated with cataract is one of the most important causes of visual impairment in ophthalmology patients, which causes visual impairment and even blindness all over the world.

[1] Unfortunately, there is a lack of research on its pathogenesis. Human lens epithelial cells(HLECs) is the most active cells of the lens. As a mediation barrier between crystal fibers and aqueous humor, HLECs play a vital role in regulating ion concentration and water accumulation so as to protect the interior of the lens. The functional change of HLECs is considered to be the cellular basis of cataract development, which is closely related to the formation of cataract. ${ }^{[2]}$ Besides, HLECs are close to the anterior chamber, which is the most easily affected by uveitis anterior inflammation, resulting in cataracts.

Nod-like receptor heat protein domain related protein 3 (NLRP3) body is composed of procaspase1, NLRP3 and ASC. After the self-activation of procaspase-1. It promotes the release of inflammatory cytokines IL-1ßand IL-18, further aggravating the inflammatory response. NLRP3 inflammasome plays a role in the development of diabetic retinopathy, age-related cataract and other diseases. ${ }^{[3-4]}$ However, whether NLRP3 inflammasome is expressed in the anterior capsule of uveitis associated with cataract has not been investigated in ophthalmology. Apoptosis of epithelial cells can promote the formation of cataracts $^{[5]}$. Although numerous experiments confirm this process, there are little intuitive evidence to prove it. Electron microscope observation helps us to verify the pathogenesis.

In this study, we reserached the expression of NLRP3 inflammasome in lens anterior capsule of uveitis associated with cataract using the anterior capsule. Immunohistochemistry and electron microscope were used to observe the expression of NLRP3 inflammasome and ultrastructure changes in lens anterior 
capsule. Our studies have explored the NLRP3 expression and significative ultrastructure changes in the anterior capsule, which suggests a new novel mechanism underlying the disease pathogenesis.

\section{Methods}

Subject 17 cases (22 eyes) of patients with cataracts associated with uveitis in the ophthalmic inpatient department of our hospital were randomly selected as the experimental group and 10 cases (18 eyes) ofage-related cataract patients as the control group from August 2018 to June 2019. All the anterior capsule were obtained on posterior continuous curvilinear capsulorhexis. The factors such as diabetes, organ injury, hypertension and age, nature of work, living environment, severity of illness, gender and living habits were excluded. Ophthalmic examination excluded other eye diseases, and none of the above cases had a long history of medication. All eyes were > for 3 months before operation.

Immunohistochemical Human lens epithelial tissues were removed and immediately embedded by a paraffin embedding machine. The embedded specimens were pre-cooled in liquid nitrogen for $2 \mathrm{~min}$, and then stored in a low-temperature refrigerator at $-80^{\circ} \mathrm{C}$. The paraffin-embedded sections dewaxed by xylene were soaked in alcohol and water for the hydration and soaked in PBS. Following that, the antigen repair was performed under the high pressure. The slices were immersed in water, poured into EDTA repair solution, boiled and repaired about $2 \mathrm{~min}$. After cooling, $3 \% \mathrm{H} 2 \mathrm{O} 2$ was added to continue the incubation at room temperature for $20 \mathrm{~min}$. After that, the sections were rinsed 3 times with PBS and dried, and the primary antibody was added to incubate at $37^{\circ} \mathrm{C}$ for $60 \mathrm{~min}$. The slices were then washed off 3 times and dried. Following that, the secondary antibody was added to incubate $30 \mathrm{~min}$. Then, the slices were washed with PBS three times and stained by DAB method. Image ProPlusV6.0 was adopted to analyze the protein expression of NOTP, ASC and Caspase1 by optical density analysis.

Ultrastructure under the electron microscope The anterior capsule membrane was stored in glutaraldehyde at $5{ }^{\circ} \mathrm{C}$ for 24 hours. Afterwards, the membrane was rinsed in PBS buffer solution and fixed in $1 \%$ osmium tetroxide. Following that, the membrane washed off was dehydrated by ethanol, fixed and embedded. Further, the repaired embedded block was sliced into ultra-thin sections, with a thickness of 70nm and taken out by a copper mesh. Ultimately, the sections were stained with electron (lead and uranium) and observed under an electron microscope.

Statistical analysis Statistical analysis was performed using the SPSS 20.0 statistical software. All data were expressed as mean \pm standard deviation (SD). Differences were analyzed by paired t-test and ANOVA and considered statistically significant at $\mathrm{P}<0.05$.

\section{Results}


Caspase-1 expression: the expression of caspase-1 in the lens anterior capsule of the lens in uveitis complicated with cataract (Figure $1 \mathrm{~A}$ ) was significantly higher than that in the lens anterior capsule of the lens in age-related cataract (Figure $1 \mathrm{D})$, and the difference was statistically significant $(P<0.05)$.

ASC expression: the expression of ASC in the lens anterior capsule of lens in uveitis complicated with cataract (Figure $1 \mathrm{~B}$ ) was significantly higher than that in the lens anterior capsule of lens in age-related cataract (Figure $1 \mathrm{E})$, and the difference was statistically significant $(P<0.05)$.(table 2 for data)

NLRP3 expression: NLRP3 is positively expressed in lens anterior capsule of lens inage-related cataract and uveitis complicated with cataract, but NLRP3 expression is significantly higher in lens anterior capsule of lens in uveitis complicated with cataract than in lens anterior capsule of lens in age-related cataract (Figure $1 \mathrm{C}$ and Figure $1 \mathrm{~F}$ ), the difference is statistically significant $(P<0.05)$;

Table 1 The comparison of optical density values of NLRP3, caspase-1 and ASC in the two groups $\rrbracket^{\prime} \mathrm{x} \pm \mathrm{S} \llbracket \mathrm{OD} \square$

\begin{tabular}{|c|c|c|}
\hline Group & 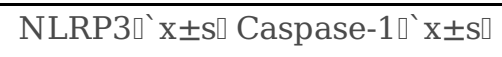 & $\overline{A S C} \llbracket{ }^{\prime} \mathrm{x} \pm \mathrm{S} \square$ \\
\hline \multicolumn{3}{|c|}{ The experimental group $0.384 \pm 0.067 \mathrm{a}_{0.321 \pm 0.057} \mathrm{a}_{0.337 \pm 0.037} \mathrm{a}$} \\
\hline The control group & $0.331 \pm 0.085 \quad 0.270 \pm 0.044$ & $0.259 \pm 0.038$ \\
\hline t value & 2.222 & 6.562 \\
\hline$P$ value & 0.032 & 0.000 \\
\hline
\end{tabular}

Note: a means $\mathrm{P}<0.05$

\section{Ultrastructure observation of lens anterior capsule of lens in uveitis associated cataract and age-related cataract under electron microscope}

In the lens epithelial cells of patients with uveitis complicated with cataract, obvious nuclear pyknosis was observed, chromatin was condensed and edge set, cell nuclear membrane was folded and slightly widened, mitochondrial morphology was basically normal, part of mitochondrial crest disappeared. In the nucleus of lens epithelial cells of age-related cataract, chromatin concentration and marginal collection were also observed. Nuclear pyknosis was mild compared with the experimental groupand mitochondrial morphology and mitochondrial crest are in normal.

\section{Discussion}

In the lens, previous studies indicate that oxidative stress conducts a pivotal role in the occurrence and progress of age-related cataracts ${ }^{[6]}$. Moreover, the abnormality of lens epithelial cells is the basis of the progress of cataract. At present, oxidative stress induced by $\mathrm{H} 2 \mathrm{O} 2$ can promote the formation of cataract. ${ }^{[5]}$ Furthermore, oxidative stress can facilitate the activation of NLRP3 that leads to processing and secretion of the proinflammatory cytokines IL-1b and IL-18. Increasing studies have found that the use of $\mathrm{H} 2 \mathrm{O} 2$ to induce oxidative stress can accelerate the activation of IL-1 $\beta$ mediated by NLRP3, thereby 
promoting the development of cataracts. ${ }^{[7-8]}$ Our study proved that NLRP3 was expressed in lens epithelial cells of age-related cataract, suggesting that it may conduct a role in promoting the development of age-related cataract.

The pathogenesis of uveitis remains unclear, but it is believed that the most common cause of inflammation is autoimmune response.In addition, the uveitis has been confirmed to be related to a variety of cytokines, including IL-1, IL-6, IL-8 and IL-23, among which IL-1 $\beta$ is mainly derived from NLRP3. Therefore, NLRP3 inflammasome has been verified to be related to the occurrence and progress of uveitis. ${ }^{[9-10]}$ What's more, IL-1 $\beta$ can promote the production of VEGF. The content of VEGF was increased in aqueous humor and serum of patients with cataract. ${ }^{111}$ XOMA 052 anti-IL-1 $\beta$ antibody can effectively treat acute and aggravated drug-resistant Behcet uveitis and IL-18 is related to the occurrence of Behcet and disease activity. ${ }^{[12]}$ In the aqueous humor with HLA-B27-related uveitis, the content of IL-18 was increased and correlated with aqueous flare. ${ }^{[13-14]} \mathrm{HLECs}$ is close to the anterior chamber and iris. IL-1 promotes leukocyte recruitment and might initiate apoptosis as a potent extracellular stimulator ${ }^{[15]}$. In this study, it was found that the expression of NLRP3 in anterior capsule of cataract patients was higher than that of senile cataract. It was speculated that HLECs was close to the anterior chamber and iris, and NLRP3 in aqueous humor of uveitis patients may transmit exogenous IL-1 $\beta$ through aqueous humor to stimulate HLECs, thereby accelerating the cataract. But this mechanism needs further experimental confirmation.

It was found that the apoptosis of lens epithelial cells was involved in the mechanism of cataract patients with exfoliation syndrome. ${ }^{[16]}$ The apoptosis-related proteins were abnormally expressed in lens anterior capsule of age-related cataract. ${ }^{[17]}$ Moreover, apoptotic lens epithelial cells damaged the function of organelle, which affected the barrier protective effect of regulating ion and water accumulation. In this study, it was found that under an electron microscope the apoptosis of lens epithelial cells in patients with complicated cataract was more obvious than that in patients with senile cataract, accompanied by the high expression of NLRP3, which may be related to the fact that the high concentration of NLRP3 promoted the apoptosis in patients with cataract.

The pyroptosis is a kind of rapid inflammatory cell death, which is characterized by the early formation of cytomembrane pore and cell permeability expansion. ${ }^{[18]}$ Besides, the apoptosis is the main mode of cell death, which is considered to be immune silencing and maintains the completeness of cytomembrane until the cell is engulfed. It's worth noting that NLRP3 plays a crucial role in both the pyroptosis and apoptosis. ${ }^{[19]}$ AIM2(absent in melanoma-2) and NLRP3 activate caspase- 8 and caspase-1, leading to apoptosis and pyroptosis.Additionally, the balance between apoptosis and pyroptosis depends on the concentration of DNA. Given that the pyroptosis may also be one of the mechanisms of cataract ${ }^{[20]}$, NLRP3 may promote the development of cataract both by the apoptosis and pyroptosis. The apoptosis dependent on caspase- 1 is first initiated through ASC, and ASC is necessary for all caspase fragmentation. Besides, ASC is a key molecule involved in the synthesis of procaspase- 1 and procaspase8 and plays an important role in the pyroptosis and apoptosis. ${ }^{[19]}$ It is not difficult to understand the high 
positive expression of ASC in anterior capsule of the cataract. The pyroptosis features the obvious swelling of cells and formation of cell pores. There are little reports on ultrastructural changes of anterior capsule cells in patients with cataract under electron microscope, and the further study is needed in the future.

ATP and intracellular $\mathrm{K}$ ion efflux can induce the activation of NLRP3 inflammasome, both of which are abundant in cells. ${ }^{[21-22]}$ NLRP3 can promote the pyroptosis by activating Caspase-1, which can damage the plasma membrane and expose the contents of cytoplasm. By this manner, ATP and potassium ions will flow out with the process of pyroptosis, thus further activating NLRP3 inflammasome. This mechanism may be another reason for the high positive expression of NLRP3 inflammasome.

This research was limited due to the sample size enrolled in this experiment is relatively small result from the limited number of cases.

\section{Conclusion}

During the development of Uveitis associated with cataract, the expression of NLRP3 inflammasome increases. Generally, the apoptosis is a vital feature of anterior capsule changes in patients with cataract. Hence, our data indicate that NLRP3 inflammasome may be involved in the development of cataract by promoting apoptosis. It's imperative to collect a larger sample to further validate our results. Complex and multiple factors is involved in the development of uveitis associated with cataract, and its underlying mechanism remains far from being fully clarified. This study can provide new direction in its reserach and treatment of preventive measures.

\section{Abbreviations}

NLRP3: nod-like receptor pyrin domain 3

ASC: Apoptosis-related speckle protein

HLECs: Human lens epithelial cells

AlM2: Absent in melanoma-2

\section{Declarations}

\section{Ethics approval and consent to participate}

The study was approved by Ethics Committee of Anhui Provincial Hospital of Anhui Medical University and was conducted in accordance with the Declaration of Helsinki. All patients voluntarily signed the informed consent.

\section{Consent for publication}


Not applicable.

\section{Availability of data and materials}

The datasets during and/or analyzed during the current study are available from the corresponding author on reasonable request.

\section{Competing interests}

The authors declare that they have no competing interests.

\section{Funding}

Natural Science Foundation of Anhu Province 1508085 MH188 8 Education Department of Anhui Province Teaching research project囚2016jyxm0546》

\section{Authors' contributions}

Zi-Cheng Zhu and Chu Zhang were primarily conceived experimental concept and design. Chu Zhang participated in information gathering and editing. Zi-Cheng Zhu was also involved in data analysis. All authors reviewed and approved the final manuscript.

\section{Acknowledgements}

None.

\section{References}

[1]Nussenblatt RB. The natural history of uveitis[J]. Int Ophthalmol 1990;14(5-6):303-308.

[2] Li D, Liu GQ, Lu PR, High glucose: activating autophagy and affecting the biological behavior of human lens epithelial cells[J]. Int J Ophthalmol 2019;12(7)囚1061-1066.

[3] Doyle SL1, Campbell M, Ozaki E,et al.NLRP3 has a protective role in age-related macular degeneration through the induction of IL-18 by drusen components[J]. Nat Med. 2012 May;18(5):791-798.

[4] Devi TS1, Lee I, Hüttemann M,et al. TXNIP links innate host defense mechanisms to oxidative stress and inflammation in retinal Muller glia under chronic hyperglycemia: implications for diabetic retinopathy[J].Exp Diabetes Res. 2012;2012:1-19.

[5] Y. W. Mao, H. Xiang, J. Wang, et al. Human bcl-2 gene attenuates the ability of rabbit lens epithelial cells against $\mathrm{H} 2 \mathrm{O} 2$-induced apoptosis through down-regulation of the alpha B-crystallin gene[J]. J Biol Chem 276(2001) 43435-43445. 
[6] Berthoud VM, Beyer EC. Oxidative stress, lens gap junctions, and cataracts[J]. Antioxid Redox Signal. 2009 Feb;11(2):339-53.

[7] Marneros AG.NLRP3 inflammasome blockade inhibits VEGF-A-induced age-related macular degeneration[J]. Cell Rep 2013.4: 945 - 958.

[8] Marneros AG.Increased VEGF-A promotes multiple distinct aging diseases of the eye through shared pathomechanisms[J]. EMBO Mol Med.2016 8(3):208-31.

[9] González-Benítez JF, Juárez-Verdayes MA, Rodríguez-Martínez S, et al. The NALP3/ Cryopyrin-Inflammasome complex is expressed in LPS-induced ocular inflammation[J]. Mediators Inflamm, 2008, 2008: 1-7.

[10] Wan CK, He C, Sun L, et al. Cutting edge: IL-1 receptor signaling is critical for the development of autoimmune uveitis [J]. J Immunol, 2016, 196(2): 543-546. DOI: 10. 4049/ jimmunol. 1502080.

[11] Paroli MP, Teodori C, D'Alessandro M, Mariani P, lannucci G, Paroli M.Increased vascular endothelial growth factor levels in aqueous humor and serum of patients with quiescent uveitis [J]. Eur $\mathrm{J}$ Ophthalmol. 2007 Nov-Dec;17(6):938-942.

[12] Gül A,Tugal-Tutkun I,Dinarello CA, et al. Interleukin-1ß-regulating antibody XOMA 052 (gevokizumab) in the treatment of acute exacerbations of resistant uveitis of Behcet's disease: an open-label pilot study[J]. Ann Rheum Dis.2012 71(4):563-6.

[13] Musabak U, Pay S, Erdem H, Simsek I, Pekel A, Dinc A, Sengul A.Serum interleukin-18 levels in patients with Behçet's disease. Is its expression associated with disease activity or clinical presentations? [J]. Rheumatol Int. 2006 Apr;26(6):545-550.

[14] Zhao B, Chen W, Jiang R.et al. Expression profile of IL-1 family cytokines inaqueous humor and sera pf patients with HLA -B27 associated[J].Exp Eye Res. 2015 Sep; 138:80-86.

[15] Clarke MC, Talib S, Figg NL, Bennett MR. Vascular smooth muscle cell apoptosis induces interleukin1-directed inflammation: effects of hyperlipidemia-mediated inhibition of phagocytosis. Circ Res 2010; 106: 363-372.

[16]Oltulu P , Oltulu R. The Association of Cataract and Lens Epithelial Cell Apoptosis in Patients with Pseudoexfoliation Syndrome[J]. Curr Eye Res. 2018 Mar;43(3):300-303.

[17] Li G , Song H , Chen L, et al, TUG1 promotes lens epithelial cell apoptosis by regulating miR421/caspase-3 axis in age-related cataract[J]. Exp Cell Res. 2017 Jul 1;356(1):20-27.

[18] Fink SL, Cookson BT. Caspase-1-dependent pore formation during pyroptosis leads to osmotic lysis of infected host macrophages[J].Cell Microbiol 2006; 8: 1812-1825. 
[19] Sagulenko V, Thygesen SJ, Sester DP,et al, AIM2 and NLRP3 inflflammasomes activate both apoptotic and pyroptotic death pathways via ASC[J]. Cell Death Differ.2013 20(9):1149-60.

[20] Jin X, Jin H, Shi Y ,et al, Pyroptosis, a novel mechanism implicated in cataracts[J]. Mol Med Rep. 2018 Aug;18(2):2277-2285.

[21] Karmakar M,Katsnelson MA, Dubyak GR, et al, Neutrophil P2X7 receptors mediate NLRP3 inflammasome-dependent IL-1 $\beta$ secretion in response to ATP[J]. Nat Commun. 2016 Feb 15;7:10555.

[22] Katsnelson MA, Rucker LG, Russo HM, et al, K+ efflux agonists induce NLRP3 inflammasome activation independently of Ca2+ signaling[J]. J Immunol. 2015 Apr 15;194(8):3937-52.

\section{Figures}
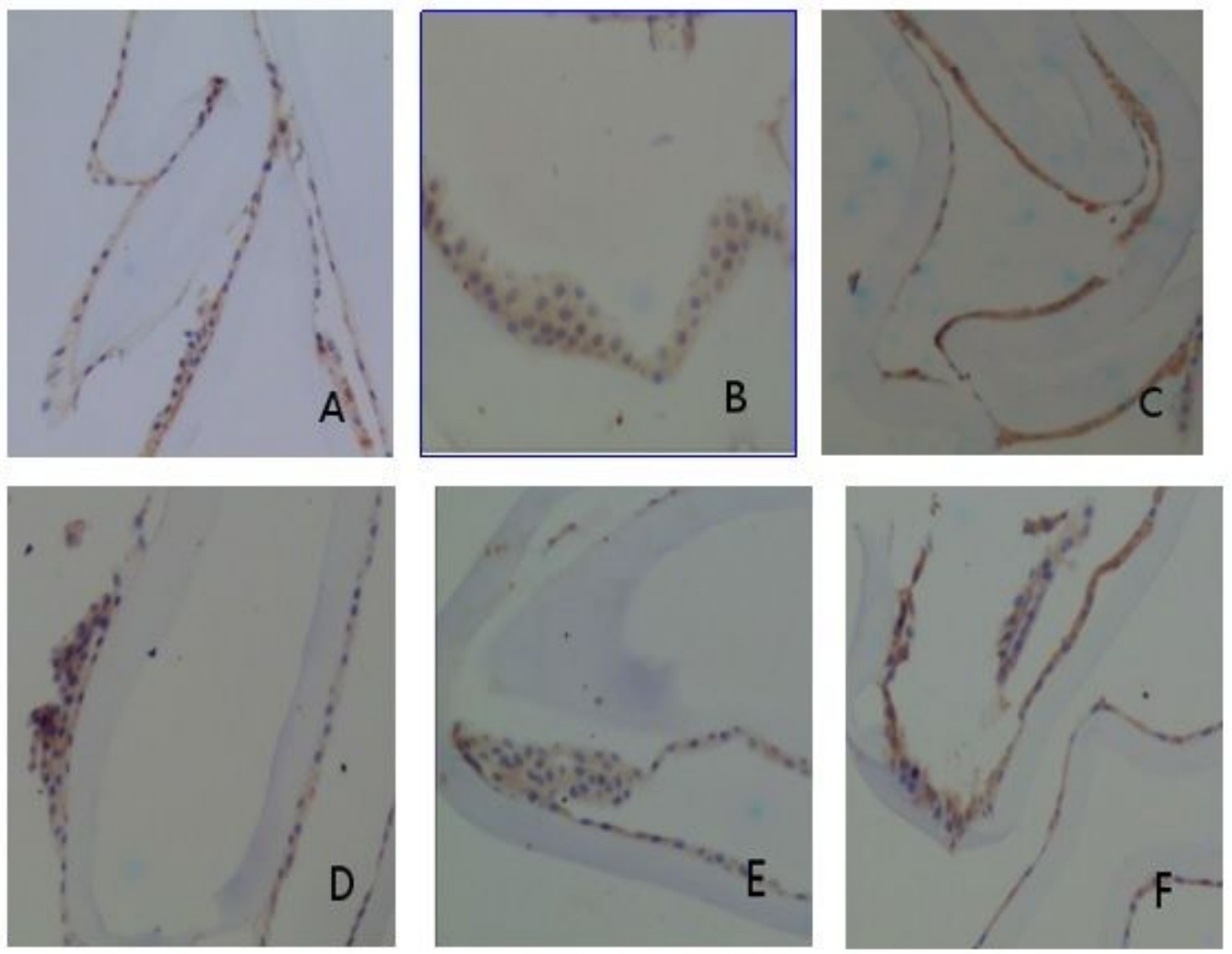

\section{Figure 1}

The expression of NLRP3 inflammasome in lens anterior capsule of each group detected by immunohistochemistry. A Expression of caspase-1 can be seen in the experimental group. B Expression of ASC can be seen in the experimental group. C Expression of NLRP3 can be seen in the experimental group. D Expression of caspase-1 can be seen in the control group,lower than that in the group A. E 
Expression of ASC can be seen in the control group,lower than that in the group B. F Expression of NLRP3 can be seen in the control group,lower than that in the group $\mathrm{C}$.
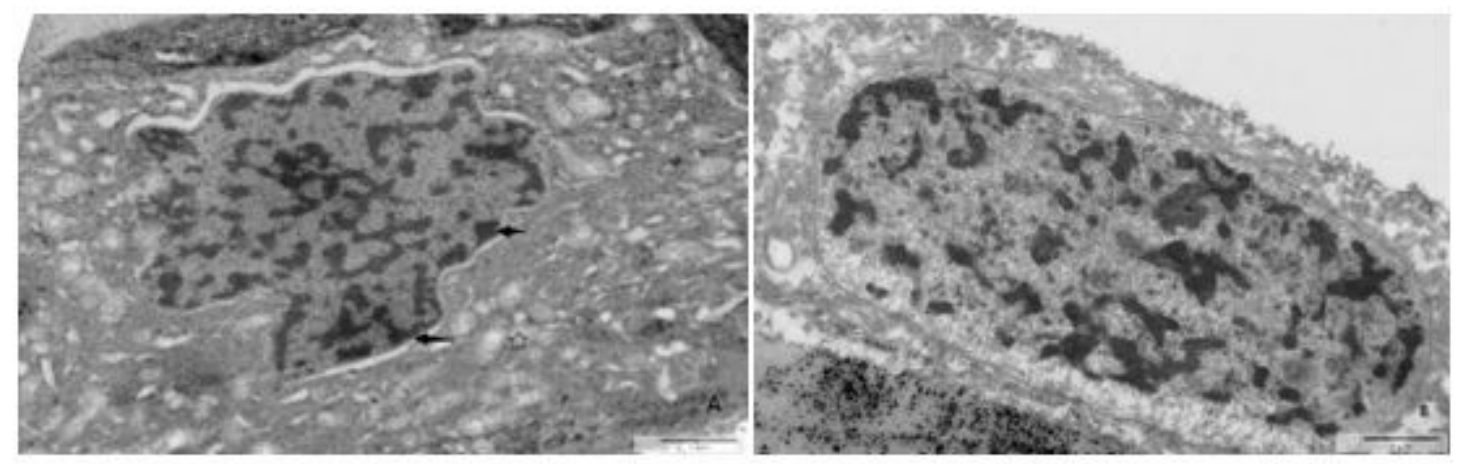

Figure 2

Ultrastructure changes in lens anterior capsule of each group A: apoptotic changes in lens epithelial cells are seen in patient with uveitis associated with cataract, with chromatin condensed and edge set (Indicated by the arrows) \part of mitochondrial crest disappeared》Indicated by an asterisk囚. B: apoptotic changes were also observed in the control group, but some changes were mild compared with the experimental group 